\title{
Preparing for the Certification and Recertification Examination
}

One of the most frequent questions we are asked at the offices of the American Board of Family Practice is "How can I prepare for the certification or recertification examination?" The answer is complex; there is no straightforward, simple way to answer it. All the questions on the certification and recertification examinations are referenced so we can identify where the information came from and why the answer was selected. Reviewing these references, you would find that most of the questions and answers come from standard texts and the current literature.

We have always felt the best preparation for these examinations is to be actively involved in the full breadth of family practice; to read the current literature; to attend clinic, staff, and hospital conferences; and to attend regularly those continuing medical education (CME) programs that are focused on areas of need, not just areas of special interest. A great number CME programs and board reviews are offered by educational institutions and the American Academy of Family Physicians, many of which can be helpful when preparing for the examination.

The ABFP reference guides and in-training assessment examination questions are available at www.familymed.com. Although the specific questions found there will not be used in future examinations, reviewing them can help prepare for the types of questions that will be on the examination.

The purposes of this article are to provide a general description of the item and test development process so that the candidate can understand how the ABFP develops, evaluates, and selects test items for examinations, and to offer some suggestions for preparation and test-taking strategies.

\section{Development of the Examination}

All questions on the certification and recertification examinations are written by family physicians who are either in private practice or are affiliated with family practice residency programs. The initial phase of the examination is the submission of test questions by $\mathrm{ABFP}$-trained item writers who have attended board-sponsored workshops for writing examination questions. Each item writer is asked to submit 20 questions each year assigned on a quarterly basis. Currently, there are 35 practicing family physicians who write test questions.

Each question submitted is accompanied by documentation from a current and reliable journal or an accepted up-to-date textbook. The purpose of documentation is to justify the correct answer. A member of the test development staff reviews these submitted items extensively for format, grammar, spelling, and punctuation and then enters them into a pool of items. A committee of 10 to 12 family physicians from varied backgrounds examines the questions further. Some of these physicians are in private solo practice, some in group practice, some in teaching institutions, and so on.

When it is time to create the first draft of an upcoming examination (the entire examination production process take approximately 9 to 10 months), the editors choose items from the pool according to a preset mix of question types (onebest answer, multiple true or false, and clinical sets). A predetermined percentage of items are drawn from eight disciplines. The breakdown by discipline is based on the approximate amount of time physicians spend on various disciplines in a clinical setting: $36 \%$ internal medicine, $6 \%$ surgery, $7 \%$ obstetrics, $9 \%$ community medicine, $13 \%$ pediatrics, $7 \%$ psychiatry, $12 \%$ geriatrics, and $10 \%$ gynecology.

A review committee meets three times to review each of the questions within these disciplines. The committee sees one-half again as many questions as are needed for the examination and makes judgments based on the accuracy and relevancy of each question. Some questions are eliminated during this process. After the review committee has finished, the test development staff carefully goes over each question to make certain that any changes made by the committee are clear and supported by appropriate documentation. The corrected questions and answers are then again reviewed by the staff. 
A second draft is prepared by the test development staff. Ten family physicians are selected to set the standard score (Angoff method) by verifying the correct answer to each question and estimating the percentage of marginally competent candidates who will answer it correctly. The test development editors review these questions and prepare a third draft. This final draft is reviewed by the ABFP Board of Directors. If at this time the Board sees any question that is inaccurate, out-of-date, or not relevant, they will eliminate that question.

After the final committee has met and approved the examination questions, usually in early March, the test development staff prepares the examination for printing. There are nine test booklets, including one with a pictorial insert, that are created for the examinations. These booklets must be printed, bound, shrink wrapped, and delivered to the Board office no later than June 15. During the third week of June, the examination booklets are assembled and shipped to the proctors at various test sites. The examination is given on the second Friday in July.

\section{The Day of the Examination}

All candidates (certification and recertification) take Book $A$ in the morning. The first section of Book A contains a combination of 130 one-bestanswer and multiple true-or-false questions. The second section of Book A is made up of clinical set problems, which are diagnosis- and managementoriented questions based on a clinical setting. The candidates make decisions (true-or-false format) regarding both diagnosis and management. This section has 220 scoreable options.

In the afternoon session of the examination, the certification candidates take Book $\mathrm{C}$, which is made up of 180 questions, including approximately 30 to 35 pictorial items. This book also contains a combination of one-best-answer and multiple true-orfalse questions.

The recertification candidates take three modular examinations, each made up of 60 questions. The recertification candidates select the three modular examinations from a choice of the following specialty areas: internal medicine, surgery, obstetrics, pediatrics, geriatrics, gynecology, and emergent and urgent care. They are given this choice so that their performance might better reflect the nature of their individual practices.
After the examination, the proctors return all test booklets, answer sheets, and any other examination-related material to the Board on Monday after the examination. Staff members inspect all test material. Each answer sheet is electronically scanned, counted, and checked for missing identification data. An omission report is generated so the staff members can check any answer sheet that has any answer omitted. Every effort is made to ensure each candidate has been given every chance for a maximum score.

\section{Preparing for the Examination}

The best preparation is "keeping up" through an active practice, peer interaction, $\mathrm{CME}$, review courses, and journals. All the questions are referenced from the current medical literature, which includes such journals as The fournal of the American Board of Family Practice, Archives of Family Practice, The Journal of Family Practice, 7AMA, New England Fournal of Medicine, and American Family Physician. Questions are also referenced to standard texts, such as; Rakel's Textbook of Family Practice or Taylor's Family Medicine: Principles and Practice, Harrison's Principles of Internal Medicine or Cecil Textbook of Medicine, Williams Obstetrics, and Nelson Textbook of Pediatrics. By reading current journals regularly and reviewing content areas in standard texts, candidates will help themselves be better prepared for the examination.

Candidates should choose areas of CME where preparation will help fill in gaps in their practice. Board review courses can be helpful for overall preparation. In-training examination questions are available on the Internet for review (www.familymed.com); these questions should be used only to regain familiarity with test-taking procedures and to determine areas of weakness. No currently used examination questions are available anywhere, so memorizing questions and answers is not helpful.

In addition to educational preparation, candidates should be physically and mentally ready for the examination by being rested, focused, and prepared. Theoretically, an examination score is an estimate of the test taker's knowledge of the content being assessed. This estimate of knowledge, however, might not be accurate under certain circumstances, such as when a test taker is not physically up to taking the examination, does not carefully listen to the proctor or read and follow the test directions accurately, or fails to ascertain the num- 
ber of items that must be answered and the total amount of time in which the test must be completed.

\section{Test-Taking Strategies}

As previously indicated, the ABFP certification and recertification examinations consist of three basic item formats: one best answer, multiple-choice; multiple true or false; and clinical set problems.

\section{Multiple-Choice Items (One Best Answer)}

The one-best-answer, multiple-choice items have a stem element that presents the assessment point typically in the form of a question. The task of the test taker is to select one option that represents the best answer to the question. Some suggested rules for taking this item format are listed below:

- Read the stem carefully and underline especially relevant information.

- Make a mental note or make notes on the test booklet.

- Read each option carefully. Underline relevant information. If you are quite sure that an option is not correct, cross out the option letter.

\section{Multiple True-or-False Items}

The multiple true-or-false items are used to assess content for which there might be more than one correct answer. Multiple true-or-false items have a stem element that presents the assessment point typically in the form of a question. The task of the test taker is to respond true or false to each of the listed options. Some suggested rules for taking this item format are listed below:

- Read the stem carefully and underline especially relevant information.

- Progress through the options from the first one to the last.

- Read each option carefully, and underline relevant information in the option.

\section{Clinical Set Problems}

The clinical set problem format consists of one or more sequenced clinical scenarios typically with tabular physical examination and laboratory data. Each of the clinical scenarios has an associated set of true-or-false options that are numbered lists of diagnostic, management, or treatment options. The task of the test taker is to respond true or false to each of the listed options. The following are some suggested rules for taking this item format:

- Read the first clinical scenario carefully and underline especially relevant information, such as age, sex, ethnic background, and previous findings.

- If there are tabular findings, then read these findings and mark or make notes for those that you believe are particularly relevant to the problem.

- Before reading the first assessment point, jot down your differential diagnosis or thoughts concerning the problem on the test booklet.

- Read the assessment point carefully, paying close attention to qualifying phrases, such as "at this time," "immediate management," and "as an initial step." The detailed and extensive review of the material that comprises the ABFP examinations results in precise language so all test takers have the same opportunity to answer the questions correctly.

- After reading the assessment point, make mental or written notes as to the actions you would take in your practice without looking at the choices provided for the problem.

- When reviewing selections concerning the administration of drugs, remember that the choice of drug, mode of administration, and dosage are all required to be appropriate to make a selection correct.

- Always determine whether general knowledge or patient-specific knowledge is being assessed.

- The clinical set problem item format provides physicians with an opportunity to use their clinical knowledge, experience, and expertise. The item test development process weeds out any information that is not relevant to the practice of family medicine. When taking the test, the test taker should therefore respond to the numbered selections in a manner that is consistent with the way he or she practices.

With respect to all questions on the examination, the following three rules should be noted by each test taker:

- These are not trick questions, so do not try to out-guess the item writers; rely on your knowledge to respond to the selections. 
- Make the maximum use of all the time available. Before turning in your booklet, be certain that you have completed every part of the examination. If you are in any doubt, ask the proctor for help.
- The ABFP does not use a correction-for-guessing formula when scoring its examinations. You therefore enhance your chances of increasing your score when you guess rather than leave the item blank. 